\title{
Isothermal molecular-dynamics calculations
}

\author{
Luis F. Rull \\ Departamento Física Teórica, Facultad de Física, Universidad de Sevilla, \\ 41012 Sevilla, Spain \\ Juan J. Morales and Francisco Cuadros \\ Sección de Fisicas, Facultad de Ciencias, Universidad de Extremadura, \\ 06071 Badajoz, Spain \\ (Received 30 January 1985; revised manuscript received 28 May 1985)
}

\begin{abstract}
We have performed long-time runs of molecular-dynamics computer simulations of a two-dimensional Lennard-Jones system, without any scaling procedure. The thermodynamic properties show spontaneous fluctuations except when the system is far from the melting zone.
\end{abstract}

In a recent paper, ${ }^{1}$ Toxvaerd studied critically a special computational technique: "isothermal-isobaric" molecular dynamics ( $T-p$ MD) (used by Abraham and $\mathrm{Koch}^{2}$ to investigate melting in a two-dimensional Lennard-Jones system). By using the calculation of the isothermal compressibility from the fluctuations of volume, Toxvaerd established that the scaling procedure was incorrect and cannot be used to obtain definitive statements about the nature of the phase transition. Toxvaerd noted that the $T-p$ MD calculations, inspired by the $N-p-T$-ensemble Monte Carlo method, are obtained from Laplace transforms of the canonical partition function, and this transformation has to be performed for constant extensive state variables, excluding the volume.

In an analysis of the ensembles used in moleculardynamics simulations, Lado $^{3}$ found that, in the classical limit, for interacting systems with hard-core interactions, Monte Carlo and molecular-dynamics calculations should yield the same equation of state without corrections, i.e.,

$$
(\beta p)_{N-V-T}=\frac{N}{V}+\frac{\partial \ln \Omega_{N-V}}{\partial V}=(\beta p)_{N-V-E},
$$

where the configurational integral $\Omega_{N-V}$ is defined by

$$
\Omega_{N-V}=V^{-N} \int d r^{N} W\left(r^{N}\right),
$$

where

$$
W\left(r^{N}\right)= \begin{cases}0, & U\left(r^{N}\right)=\infty, \\ 1, & U\left(r^{N}\right)=0,\end{cases}
$$

and, as usual, $\beta=\left(k_{B} T\right)^{-1}$, with the only assumptions being the basic postulates of equilibrium statistical mechanics.

The left-hand side $(N-V-T)$ corresponds to the Monte Carlo calculation, that is, with the number of particles, volume, and temperature constant, and the right-hand side $(N-V-E)$ corresponds to the molecular dynamics calculation, with the number of particles, volume, and energy constant.

Nose ${ }^{4}$ has recently made an exhaustive study of the ensembles in molecular dynamics to reproduce both the canonical and the isothermal-isobaric probability densities in phase space. The physical system of interest consists of $N$ particles, to which an external macroscopic variable and its

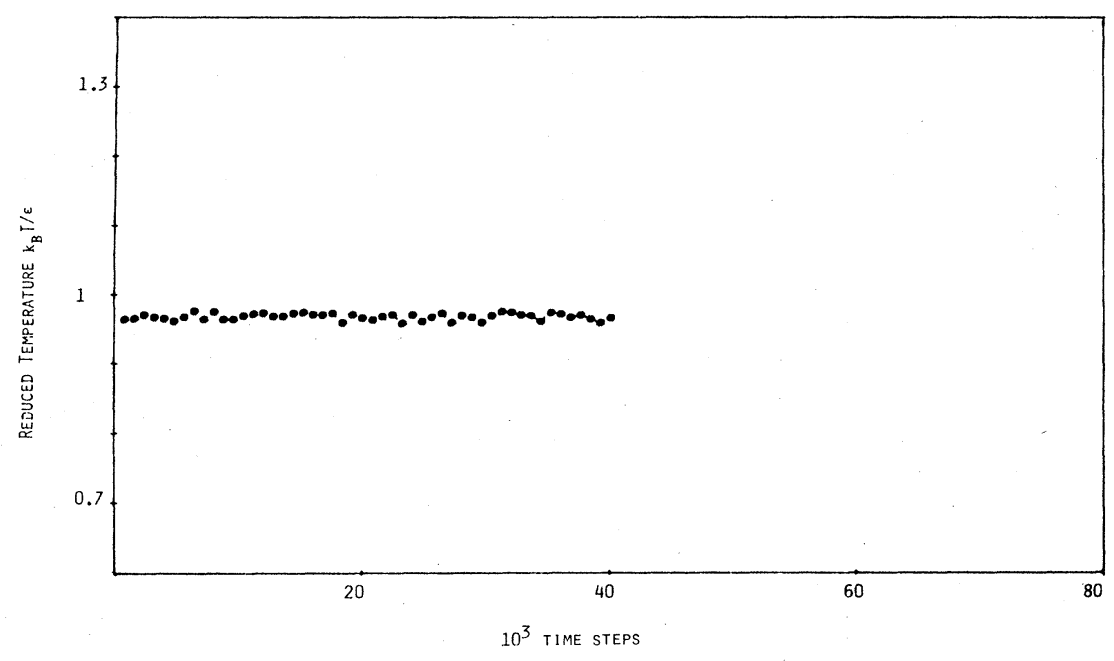

FIG. 1. Reduced temperature vs time for a two-dimensional liquid at $\rho r_{m}^{2}=1.00$. 


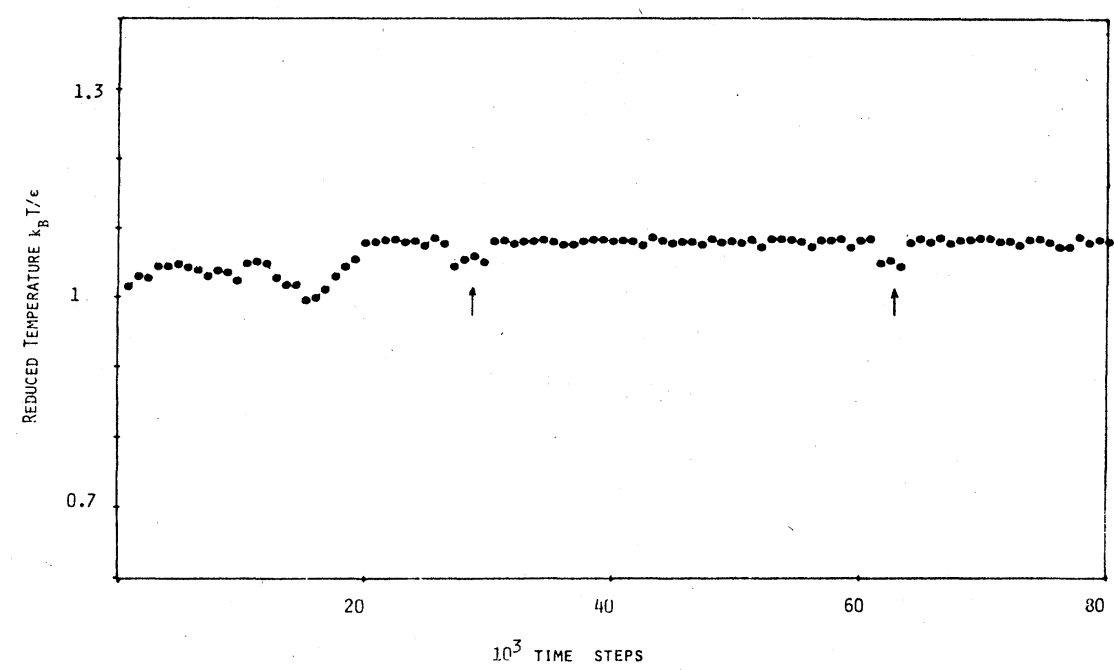

FIG. 2. Same as Fig. 1, but for a two-dimensional solid very close to the melting zone. The density is $\rho r_{m}^{2}=1.14$.

conjugate momentum are added, permitting the energy of the system to fluctuate in a way similar to Haile and Gupta's results with a thermal bath. ${ }^{5}$

In this Brief Report; we present some results of two long runs in two dimensions close to and far from the melting zone, which were performed in order to investigate the fluctuations of the thermodynamic variables during the time of evolution of the system. Our simulations have been carried out without any scaling procedures (for pressure and temperature). Just as it is incorrect to perform a simulation using the constant-pressure method in the melting zone, it is also incorrect to use the constant-temperature method, because there are problems related to fluctuations in space and time independent of the nature of the phase transition (first or second order).

In our calculations we have chosen a system of $N=256$ particles interacting with a truncated Lennard-Jones poten- tial at $r_{c}=2.5 r_{m}\left(r_{m}\right.$ is the position of the potential minimum).

The initial positions of the particles formed a twodimensional triangular lattice of densities $\rho r_{m}^{2}=1.00$ and $\rho r_{m}^{2}=1.14$. To integrate the equations of motion we used the algorithm proposed by Toxvaerd. ${ }^{6}$ This algorithm is very accurate, but introduces a drift in the energy because the equations are not reversible in time; this energy-drift problem can be solved by rescaling the velocities of the particles through the heat capacity. In our simulations this was not necessary, because the maximum drift is less than $10^{-4}$, and consequently the increment in the temperature is $k_{B} \Delta T / \epsilon \cong 10^{-7}$, and, in addition, the usual periodic boundary conditions were used.

Both systems evolved in exactly the same way. After every ten time steps (updating the table of the nearest neighbors), the velocities were renormalized to give the re-

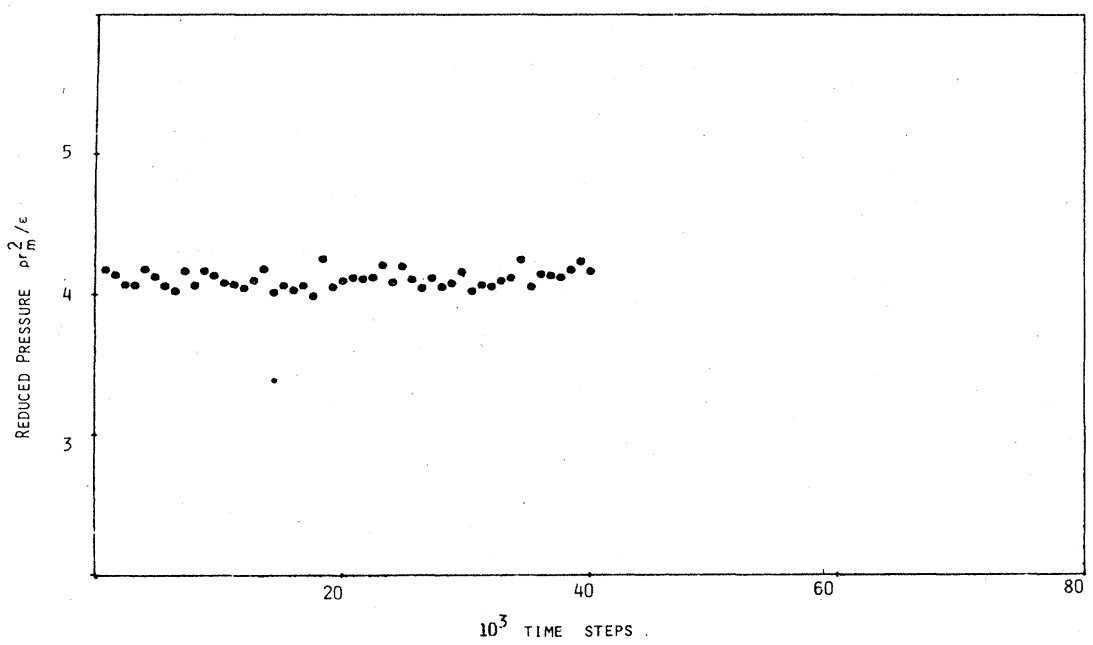

FIG. 3. Reduced pressure vs time for a two-dimensional liquid at $\rho r_{m}^{2}=1.00$. 


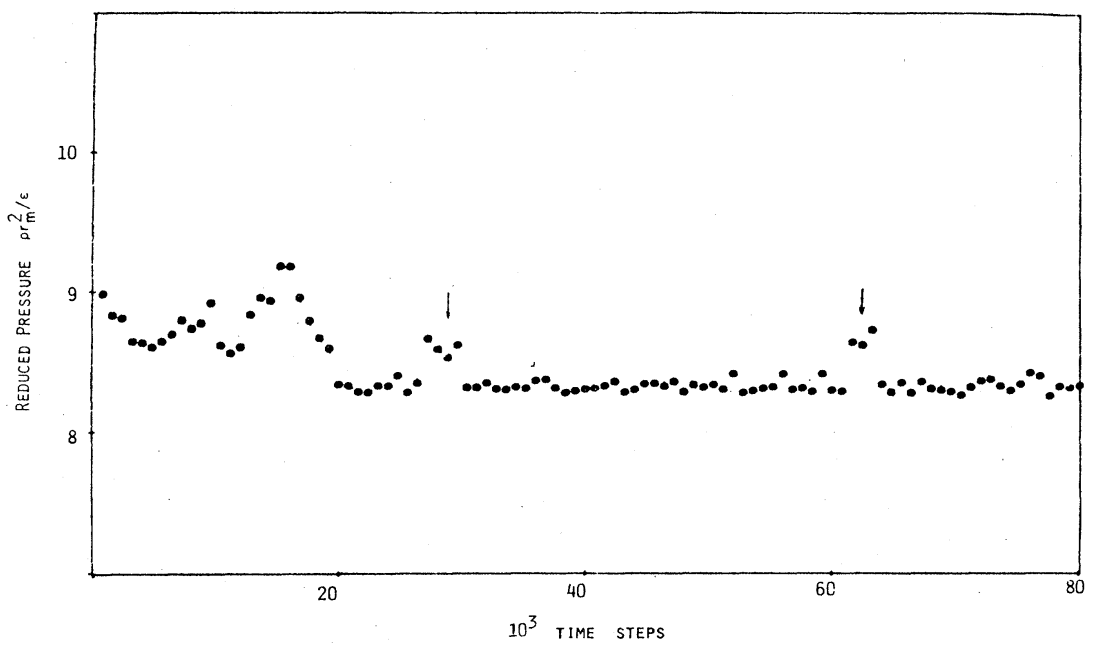

FIG. 4. Same as Fig. 3, but for a two-dimensional solid very close to the melting zone. The density is $\rho r_{m}^{2}=1.14$.

duced temperature $k_{B} T / \epsilon=1.00$ ( $\epsilon$ is the minimum of the Lennard-Jones potential). The rescaling procedure was performed during the first 8000 time steps. After that the evolution of the system was free, without any scaling procedure, the thermodynamic properties being then obtained as time averages for each 800 time steps. The virial pressure was obtained for the truncated Lennard-Jones potential, not for a full Lennard-Jones potential.

The state points that we have chosen correspond to the fluid state $\left(\rho r_{m}^{2}=1.00\right)$ and the solid state $\left(\rho r_{m}^{2}=1.14\right)$, the latter very close to the melting zone located thermodynamically by Toxvaerd. ${ }^{7}$ Toxvaerd calculated the point where fluid and solid have equal chemical potentials corresponding to the temperature $k_{B} T / \epsilon=1.00$, the difference in our case being that we have performed long runs ( 40000 time steps for a fluid and 80000 time steps for a solid).

Figures 1-4 show the results of our simulations., Each point in these figures corresponds to an average over 800 time steps. In the liquid system (Figs. 1 and 3 ) the temperature and pressure show the "regular" fluctuation typical of the liquid system, and the system is in equilibrium once free evolution commences. But for the system close to the melting zone, equilibrium is reached after 20000 time steps (Figs. 2 and 4), and the temperature spontaneously drops twice (arrows), and consequently the pressure rises.

Toxvaerd ${ }^{7}$ wondered if the equilibrium state of the system could be obtained in a time interval of 10000 time steps. Our answer is no, because in the melting zone (or close to it) the system spontaneously goes back and forth between two different points of state.

It seems evident that far from a transition in liquid or solid systems, computer simulations using the $T-p$ MC method are reliable because the fluctuations are small. However, fluctuations play an important role in the melting zone; so to obtain a definitive statement about the nature of the phase transition one should use MD simulations of free evolution, without any scaling procedure, i.e., without temperature or pressure constraints. These constraints imply that the system is not isolated, but is in contact with an energy reservoir and that the energy is transferred by a generalized force. ${ }^{5}$

The cooperation of the University of Sevilla Computer Center is gratefully acknowledged.
${ }^{1}$ S. Toxvaerd, Phys. Rev. B 29, 2821 (1984).

${ }^{2}$ F. F. Abraham and S. W. Koch, Phys. Rev. B 29, 2824 (1984), and references cited therein.

${ }^{3}$ F. Lado, J. Chem. Phys. 75, 5461 (1981).
${ }^{4}$ S. Nosé, Mol. Phys. 52, 255 (1984); J. Chem. Phys. 81, 511 (1984).

5J. M. Haile and S. Gupta, J. Chem. Phys. 79, 3067 (1983).

${ }^{6}$ S. Toxvaerd, J. Comput. Phys. 47, 444 (1982).

${ }^{7}$ S. Toxvaerd, Phys. Rev. A 24, 2735 (1981). 